\title{
Real-time Remote Reporting of Motion Analysis with Wi-FLIP
}

\author{
Jorge Fernández-Berni, Ricardo Carmona-Galán, Ángel Rodríguez-Vázquez \\ Institute of Microelectronics of Seville (IMSE-CNM), Seville, Spain \\ Consejo Superior de Investigaciones Científicas y Universidad de Sevilla
}

\begin{abstract}
This paper describes a real-time application programmed into Wi-FLIP, a wireless smart camera resulting from the integration of $F L I P-Q$, a prototype mixed-signal focal-plane array processor, and Imote2, a commercial WSN platform. The application consists in scanning the whole scene by sequentially analyzing small regions. Within each region, motion is detected by background subtraction. Subsequently, information related to that motion - intensity and location - is radio-propagated in order to remotely account for it. By aggregating this information along time, a motion map of the scene is built. This map permits to visualize the different activity patterns taking place. It also provides an elaborated representation of the scene for further remote analysis, preventing raw images from being transmitted. In particular, the scene inspected in this demo corresponds to vehicular traffic in a motorway. The remote representation progressively built enables the assessment of the traffic density.
\end{abstract}

\section{INTRODUCTION}

Image simplification is a key issue when it comes to implement a certain algorithm on vision-enabled Wireless Sensor Network (WSN) nodes [1]-[3]. It permits to alleviate the resource allocation during the processing and hence to reduce the power consumption. According to the computer vision framework [4], early vision represents the processing stage where the greatest computational effort must be done in order to attain an adequate scene representation for further understanding in subsequent stages. Alternative architectures can be proposed to handle early vision more efficiently than conventional imager-memory-DSP architectures. For example, by making the most of the ability of CMOS processes to integrate pure imaging with signal processing circuitry. Thus, the imager becomes not only an array of photosensitive devices, but an actual processing lattice composed of elementary processing cells [5] working concurrently with photosensing [6]-[8]. This is the approach supporting the design and implementation of $F L I P-Q$, a QCIF-resolution prototype chip designed ad-hoc for ultra low-power applications [9]. This chip consists of a SIMD-based mixed-signal focal-plane array implementing a subset of early vision processing primitives intended to deliver a very reduced data flow. It makes the computational load of the subsequent digital processor much lighter, reducing significantly its clock frequency and the number of memory accesses and, consequently, its power consumption. In our case, the digital processor is that of Imote 2 (MEMSIC Inc.), the commercial WSN platform which has been integrated with $F L I P-Q$ in order to obtain Wi-FLIP, a low-power visionenabled WSN node [10].

\section{REMOTE REPORTING OF MOTION ANALYSIS}

From the point of view of the energy efficiency, one of the basic operations to be implemented at a vision-enabled node is motion detection. Thorough analysis of the scene would be thus carried out only when a minimum amount of activity is taking place. But motion analysis can also be interesting by itself in scenes where such mere analysis provides really meaningful information. Consider for example a network of wireless nodes deployed along a motorway. Each node could on-site evaluate a stretch of this motorway in terms of how much activity is detected across the scene. Subsequently, the resulting information would be transmitted to a remote base station. By aggregating and merging this real-time information coming from the nodes, a remote reporting about the traffic density undergone by the motorway during a certain period of time can be easily compiled. In this demo, we address this specific application of vision-enabled motes by making use of the processing capabilities available in Wi-FLIP. A traffic scene will be continuously scanned by sequentially analyzing small regions. The location of the starting region after scanning the whole scene in each iteration will randomly change. This means that each region will be analyzed at different intervals of time, enabling to account for non-periodic motion patterns taking place within them. The analysis performed is simple. The region under study is captured and digitized a number of times by $F L I P-Q$ in each iteration. The location of such a region does not affect the processing thanks to the random pixel access scheme provided by FLIP-Q. The first snapshot captured is considered the background. The pixels $I_{F_{k l}}$ making up the following snapshots captured are compared with the corresponding pixels $I_{B_{k l}}$ of the background by means of the following equation:

$$
\left|I_{B_{k l}}-I_{F_{k l}}\right| \geq T
$$

where $T$ represents the threshold determining the sensitivity to changes at pixel level.

The intensity and location of the pixels extracted from the background by applying Eq. 1 are radio-propagated. In principle, multiple hops could occur until reaching the final destination, that is, the base station. However, for the sake of simplicity, a single hop will be implemented in this demo. Finally, a motion map is progressively built at the base station by aggregating the information coming from the node. This 

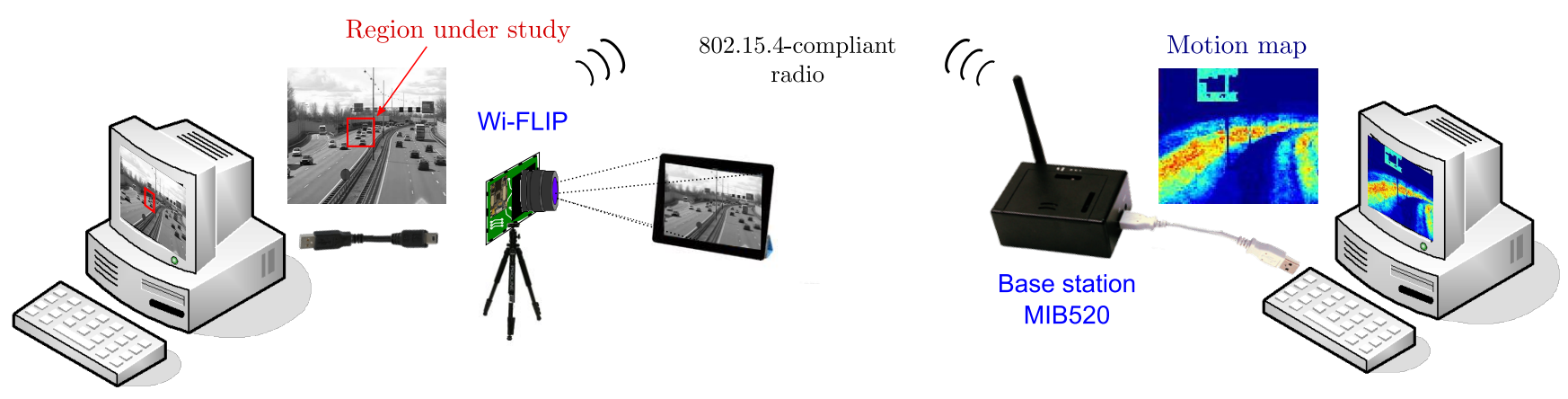

Fig. 1. General scheme of the demo.

map highlights those lanes undergoing heavy traffic, providing valuable data for a better exploitation of the motorway capacity.

\section{DEMO SETUP}

The general scheme of the demo is depicted in Fig. 1. A $n e s C$-coded Tiny OS [11] application carrying out the operation described in the previous section will run on PXA271, the 32bit ARM5 processor of the Imote2 platform. This platform will be surveying traffic footage displayed on a tablet. The progressive scanning realized by the mote during the motion analysis will be displayed in a PC connected to Imote2. Regions with a size of $22 \times 18$ px will be explored. Likewise, the process of how the base station progressively builds the motion map will be displayed in another PC. After few minutes, the pattern of traffic associated with the sequence surveyed will be clearly noticeable. To this end, we use a typical graded temperature color code where those areas with greater activity feature red color whereas the areas with less activity feature blue color.

\section{ACKNOWLEDGMENT}

This work is funded by MICINN (Spain) through project TEC2009-11812, co-funded by the European Regional Development Fund, by the Office of Naval Research (USA) through grant N000141110312, by the Spanish Ministry of Science and Innovation through Project IPT-2011-1625-430000 and by the Spanish Centre for Industrial Technological Development, cofunded by the European Regional Development Fund, through Project IPC-20111009.

\section{REFERENCES}

[1] M. Rahimi, R. Baer, O. Iroezi, J. Garcia, J. Warrior, D. Estrin, and M. Srivastava, "Cyclops: In situ image sensing and interpretation in wireless sensor networks," in Proc. of 3rd Int. Conf. on Embedded Networked Sensor Systems (SenSys), 2005, pp. 192-204.

[2] S. Hengstler, D. Prashanth, F. Sufen, and H. Aghajan, "Mesheye: a hybrid-resolution smart camera mote for applications in distributed intelligent surveillance," in Proc. of 6th Int. Conf. on Information Processing in Sensor Networks, 2007, pp. 360-369.

[3] A. Rowe, D. Goel, and R. Rajkumar, "Firefly mosaic: A vision-enabled wireless sensor networking system," in 28th IEEE International RealTime Systems Symposium, RTSS, 2007, pp. 459-468.
[4] R. González and R. Woods, Digital Image Processing. Prentice Hall, 2002.

[5] L. Chua and T. Roska, "The CNN paradigm," IEEE TCAS-I, vol. 40, no. 3, pp. 147-156, 1993.

[6] Z. Lin, M. Hoffman, N. Schemm, W. Leon-Salas, and S. Balkir, "A CMOS image sensor for multi-level focal plane image decomposition," IEEE Trans. Circuits Syst. I, vol. 55, no. 9, pp. 2561-2572, 2008.

[7] J. Poikonen, M. Laiho, and A. Paasio, "MIPA4k: A 64x64 cell mixedmode image processor array," in ISCAS, 2009, pp. 1927-1930.

[8] A. Lopich and P. Dudek, "A SIMD cellular processor array vision chip with asynchronous processing capabilities," IEEE Trans. Circuits Syst. $I$, vol. 58, no. 10, pp. 2420-2431, 2011.

[9] J. Fernández-Berni, R. Carmona-Galán, and L. Carranza-González, "FLIP-Q: A QCIF resolution focal-plane array for low-power image processing," IEEE J. of Solid-State Circuits, vol. 46, no. 3, pp. 669$680,2011$.

[10] J. Fernández-Berni, R. Carmona-Galán, G. Liñán Cembrano, A. Zarándy, and A. Rodríguez-Vázquez, "Wi-FLIP: A wireless smart camera based on a focal-plane low-power image processor," in IEEE/ACM Int. Conf. on Distributed Smart Cameras, 2011.

[11] P. Levis and D. Gay, TinyOS Programming. New York, NY (USA): Cambridge University Press, 2009. 